\title{
Imaging in Pancreatic Neuroendocrine Tumors
}

\author{
Savinay Kapur ${ }^{1}$ Raju Sharma ${ }^{1}$ Ankur Goyal', \\ ${ }^{1}$ Department of Radiodiagnosis, All India Institute of Medical \\ Sciences, New Delhi, India
}

\begin{abstract}
Address for correspondence Ankur Goyal, MBBS, MD, Department of Radiodiagnosis, All India Institute of Medical Sciences, Room No. 82, New Delhi 110029, India (e-mail: ankurgoyalaiims@gmail.com).
\end{abstract}

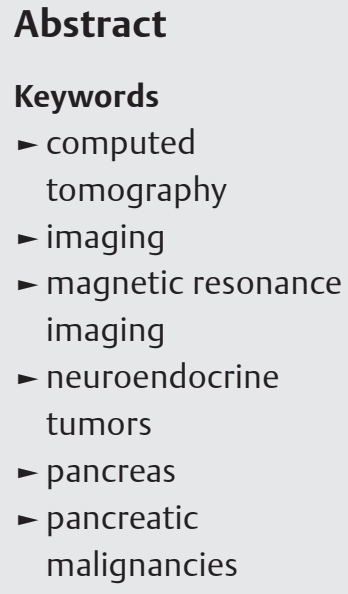

\section{Introduction}

Pancreatic neuroendocrine tumors (NET) are rare neoplasms with a reported incidence of $0.3-0.4 / 100,000$ and account for approximately 3 to $5 \%$ of all pancreatic malignancies. ${ }^{1-3}$ These tumors have a better prognosis compared with pancreatic adenocarcinomas, with an overall 5-year survival rate of $42 \%$. For resectable tumors, the 5-year survival is around $55 \%$ while for unresectable tumors, it decreases to approximately $15 \% .4,5$ These tumors may occur sporadically or in association with syndromes such as von Hippel-Lindau (vHL) syndrome, neurofibromatosis type I (NF1), multiple endocrine neoplasia type I (MEN-I), and tuberous sclerosis (TS). ${ }^{6,7}$ They arise from the islet cells of the pancreas and are similar in histology to carcinoid tumors of the gastrointestinal tract; thus, these lesions are together grouped under gastroenteropancreatic neuroendocrine tumors (GEP NETs). ${ }^{8}$ All these are epithelial neoplasms with predominant neuroendocrine differentiation. ${ }^{9}$

Imaging characterization of these lesions depends on whether the tumor is functional or nonfunctional

The challenge in imaging of functional tumors is to locate the source as these tend to be small. On the other hand, nonfunctioning tumors are large in size; thus, imaging is needed to differentiate these from other neoplasms. Another important aim of imaging is to look at dissemination and to assess resectability of these tumors. With the advent of newer nuclear medicine techniques and advancements in computed tomography (CT) and magnetic resonance imaging (MRI), it has become possible to provide accurate assessment of primary lesions as well as its spread which helps in optimizing treatment protocols. With the advent of perfusion imaging, diffusion-weighted imaging, intravoxel-incoherent motion and texture analysis, it may be possible to predict the biological behavior of these lesions. ${ }^{10-13}$

Aims of imaging in neuroendocrine tumors:

1. Detecting the presence of a hyperenhancing mass.

2. Accurate localization of the lesion.

3. Suggesting the possible diagnosis and characterize it.

4. Assessing resectability and distant spread.

5. Predicting whether the tumor will respond to therapy.

6. Early detection of residual disease or recurrence.

7. Assessing other tumors which may have a syndromic association.

\section{Imaging Techniques}

\section{Ultrasound}

Ultrasound (US) is the first imaging investigation in a patient presenting with abdominal pain.
DOI https://doi.org/ $10.1055 / \mathrm{s}-0040-1708070$ ISSN 2581-9933.
(C) 2020 by Indian Society of Gastrointestinal and Abdominal Radiology
License terms

() (1) $\ominus \circledast$ 
It may serve to detect a lesion in such symptomatic patients or rarely may detect larger lesions incidentally. However, its utility is limited as visualization of smaller focal lesions of pancreas may be obscured by bowel gas. ${ }^{14,15}$ The reported sensitivity of US for detection of GP-NETs is reported to be around 13 to $37 \% .{ }^{16}$ If visualized on US, they usually appear as hypoechoic masses with a hyperechoic halo. ${ }^{15}$ US may be more useful in visualizing and sampling metastatic liver lesions. ${ }^{17}$ Once a lesion is detected on US, CT or MRI is the next step for proper characterization and staging of these lesions.

\section{Computed Tomography}

Due to its easy availability, high spatial resolution, rapid acquisition and low cost, computed tomography (CT) has long been the workhorse of pancreatic imaging. Newer techniques such as dual-energy imaging, perfusion analysis have further added to the robustness of CT in picking up small NETs. A pancreatic protocol CT usually involves acquisition of upper abdomen in the pancreatic phase (at 35-40 s after contrast injection) followed by portal venous phase acquisition (at 60-70 s) of the entire abdomen and pelvis. Neutral oral contrast may be administered to distend small bowel loops. A noncontrast scan is not needed.

The pancreatic phase corresponds to the late arterial phase and may be acquired in dual energy mode so as to enable generation of virtual noncontrast dataset as well as for increased conspicuity of hyperenhancing lesions at low $\mathrm{keV}$ images. Usually, 80 to $120 \mathrm{~mL}$ of iodinated contrast is injected intravenously at a flow rate of 4 to $5 \mathrm{~mL} / \mathrm{s}$ followed by saline chase. All acquisition is volumetric with thin collimation. Arterial phase also helps to assess resectability by demonstrating tumor involvement of the celiac and superior mesenteric arteries and their branches.

The reported sensitivity and specificity of CT for detection of NETs is at approximately $73 \%$ and $96 \%$, respectively (-Fig. 1). ${ }^{14}$ The sensitivity is affected by the phase of acquisition, ranging from 83 to $100 \%$ in the arterial phase to 11 to $76 \%$ for portal venous phase $\mathrm{e}^{18-21}$; thus, the need of early phase. In a small study of 16 patients, evaluation of iodine material decomposition images and low $\mathrm{kV}$ monochromatic images yielded a significantly higher sensitivity of $95.7 \%$ for detection of insulinomas compared with a sensitivity of $68.8 \%$ for routine dual phase images ( - Fig. 2 ). ${ }^{22}$

\section{Magnetic Resonance Imaging}

With its multitude of sequences, magnetic resonance imaging (MRI) offers better lesion to parenchyma contrast than CT. ${ }^{10}$ It allows for lesion visualization even without administration of contrast (hence can be used in patients with renal derangement and in patients with contrast allergy) and can be safely used in young patients as there is no ionizing radiation involved. The disadvantages of MRI include higher cost, limited availability, and longer imaging times; thus, it is predominantly used as a problem-solving modality. ${ }^{10}$ The recommended MRI protocol is provided in - Table 1.

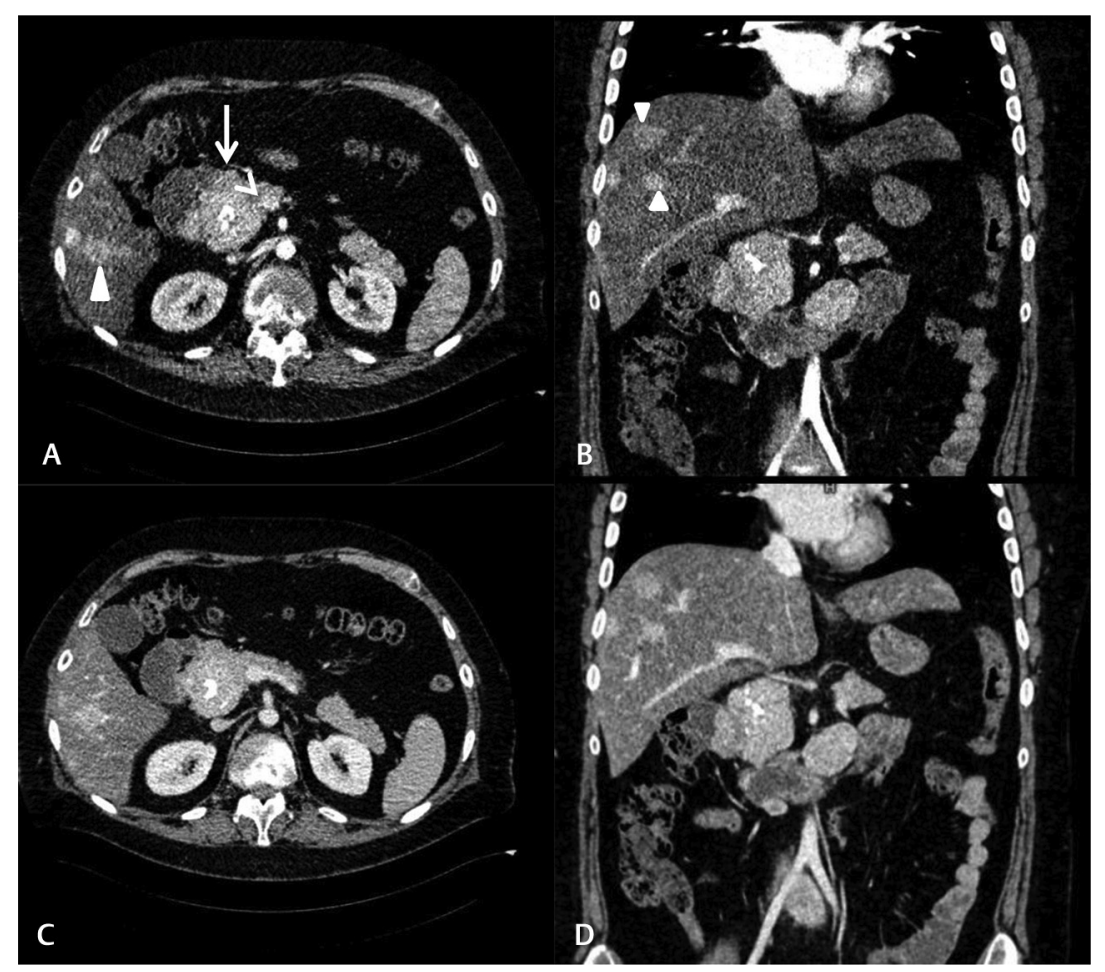

Fig. 1 (A-B) Arterial ([A] axial, [B] coronal) and venous ([C] axial, $[\mathbf{D}]$ coronal) phase images show presence of an arterial enhancing mass in the head of pancreas (arrow) with a central focus of calcification. Multiple arterial enhancing lesions are also seen in segments 6 and 8 of liver (arrowheads). Here, the lesion and metastases are well seen in both the phases. 


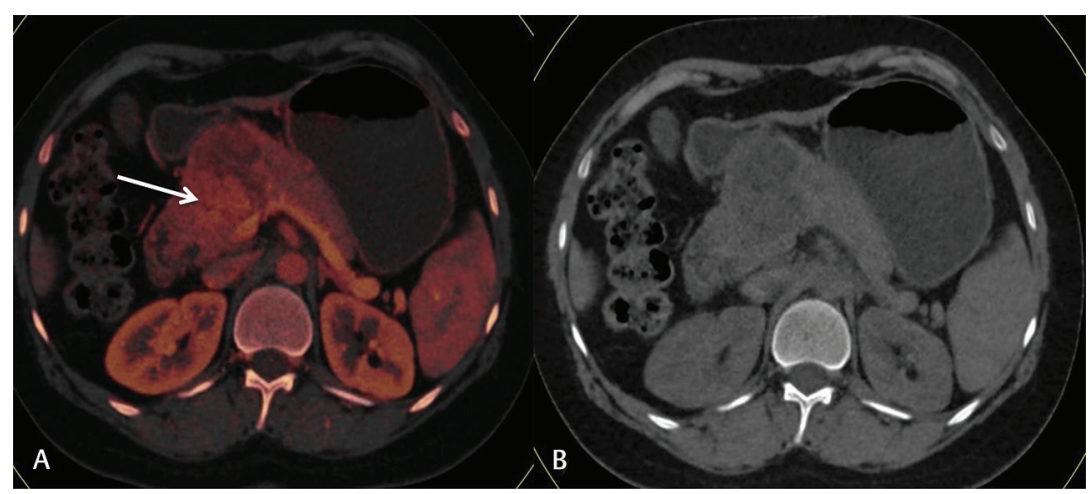

Fig. 2 Utility of dual energy CT in imaging pancreatic neuroendocrine tumors. lodine overlay map (A) and virtual noncontrast image (B) showing a solid cystic mass in the pancreatic head with definite iodine uptake in the solid component in the iodine overlay images (arrow in $\mathbf{A}$ ) in a patient with nonfunctional neuroendocrine tumor.

Table 1 Suggested MRI protocol for imaging pancreatic NETs

\begin{tabular}{|l|l|l|l|l|l|}
\hline Sequence & Matrix size & Slice thickness & TR & TE & Utility \\
\hline Axial T2-FS & $200 \times 200$ & $5 \mathrm{~mm}$ & 2,132 & 80 & \multirow{2}{*}{ NETs are hyperintense on T2 } \\
\hline Coronal T2 & $200 \times 200$ & $5 \mathrm{~mm}$ & 332 & 80 & $\begin{array}{l}\text { Fast sequence, also helps delineate relation- } \\
\text { ship with vessels }\end{array}$ \\
\hline Axial BTFE/true FISP & $200 \times 200$ & $5 \mathrm{~mm}$ & 2.7 & 1.33 & $\begin{array}{l}\text { For characterization of T1 signal and to } \\
\text { detect intracellular fat }\end{array}$ \\
\hline $\begin{array}{l}\text { Axial dual FFE / T1 in and } \\
\text { opposed phase }\end{array}$ & $188 \times 121$ & $5 \mathrm{~mm}$ & 89 & $2.3 / 4.6$ & $\begin{array}{l}\text { For assessment of pancreatic and biliary } \\
\text { ducts }\end{array}$ \\
\hline $\begin{array}{l}\text { Thick slab heavily T2W } \\
\text { MRCP }\end{array}$ & $320 \times 256$ & $40 \mathrm{~mm}$ & 8,000 & 800 & $\begin{array}{l}\text { For assessment of pancreatic and biliary } \\
\text { ducts }\end{array}$ \\
\hline $\begin{array}{l}\text { 3D RESTORE heavily } \\
\text { T2W MRCP }\end{array}$ & $125 \times 100$ & $5 \mathrm{~mm}$ & 4,365 & 64 & $\begin{array}{l}\text { Improves sensitivity of detection of primary } \\
\text { and metastatic lesions }\end{array}$ \\
\hline $\begin{array}{l}\text { Axial DWI (b-values 0 and } \\
\text { 500-800 s/mm } \text { ) }\end{array}$ & $200 \times 200$ & $3 \mathrm{~mm}$ & 5.9 & $1.80 / 4.0$ & $\begin{array}{l}\text { Arterial and venous phases are essential for } \\
\text { detection and characterization of neuroen- } \\
\text { docrine tumors }\end{array}$ \\
\hline $\begin{array}{l}\text { Dynamic pre and post } \\
\text { Gd T1 mDIXON/VIBE: } \\
\text { pancreatic, venous and } \\
\text { delayed phases }\end{array}$ & $200 \times 200$ & $3 \mathrm{~mm}$ & 5.9 & $1.80 / 4.0$ & \\
\hline $\begin{array}{l}\text { Coronal post Gd T1 } \\
\text { mDIXON }\end{array}$ & & & & \\
\hline
\end{tabular}

Abbreviations: BTFE, balanced turbo field-echo; DWI, diffusion-weighted imaging; FISP, fast imaging with steady state precession; FFE, fast field echo; MRCP, magnetic resonance cholangiopancreatography; NET, neuroendocrine tumors; VIBE, volume interpolated body examination.

The reported sensitivity and specificity of MRI for detection of neuroendocrine tumors has been reported to be $93 \%$ and $88 \%$, respectively ( - Fig. 3 ). In addition to the routine $\mathrm{T} 1$, $\mathrm{T} 2$, and arterial phase images, diffusion-weighted imaging (DWI) has been shown to improve lesion detection, differentiating intrapancreatic accessory spleen from NET as well as differentiating from other pancreatic lesions. ${ }^{23-25}$ Apart from lesion detection, MRI has also been shown to be better than $\mathrm{CT}$ in detection of liver metastasis and evaluating tumor gra de. 10,12,26 Ill-defined margins, larger size, necrosis, predominant solid component, arterial hypoenhancement, pancreatic duct dilatation, presence of metastases, and diffusion restriction were more common in grade 3 than in grade 1 or 2 NETs. ${ }^{27,28}$ In addition different DWI-based texture analysis parameters including correlation, contrast, inverse difference moment, maximum intensity, and entropy have been shown to differ between different grades of pancreatic NETs. ${ }^{27}$

\section{Nuclear Medicine}

Traditionally somatostatin receptor scintigraphy has been used for detection of NETs. This is done by using octreotide, which is an analogue of somatostatin and binds to somatostatin receptors 2 and 5 (out of the 5 types of somatostatin receptors). ${ }^{29}$ To enable its detection, octreotide is labeled with indium-111 (In-111), which emits low energy gamma photons which can be imaged using planar and single photon emission CT (SPECT) gamma cameras. Imaging is done at 4 hours and 24 hours after administration of In-111-labeled octreotide. For better localization of the tumor, a SPECT may be combined with a CT. ${ }^{30}$ The reported sensitivity of In-111 scintigraphy for detection of pancreatic NETs is reported to be around 70 to $90 \%$. Its sensitivity is significantly reduced for subcentimeter lesions and for insulinomas which express the type 3 somatostatin receptors. ${ }^{30,31}$ 


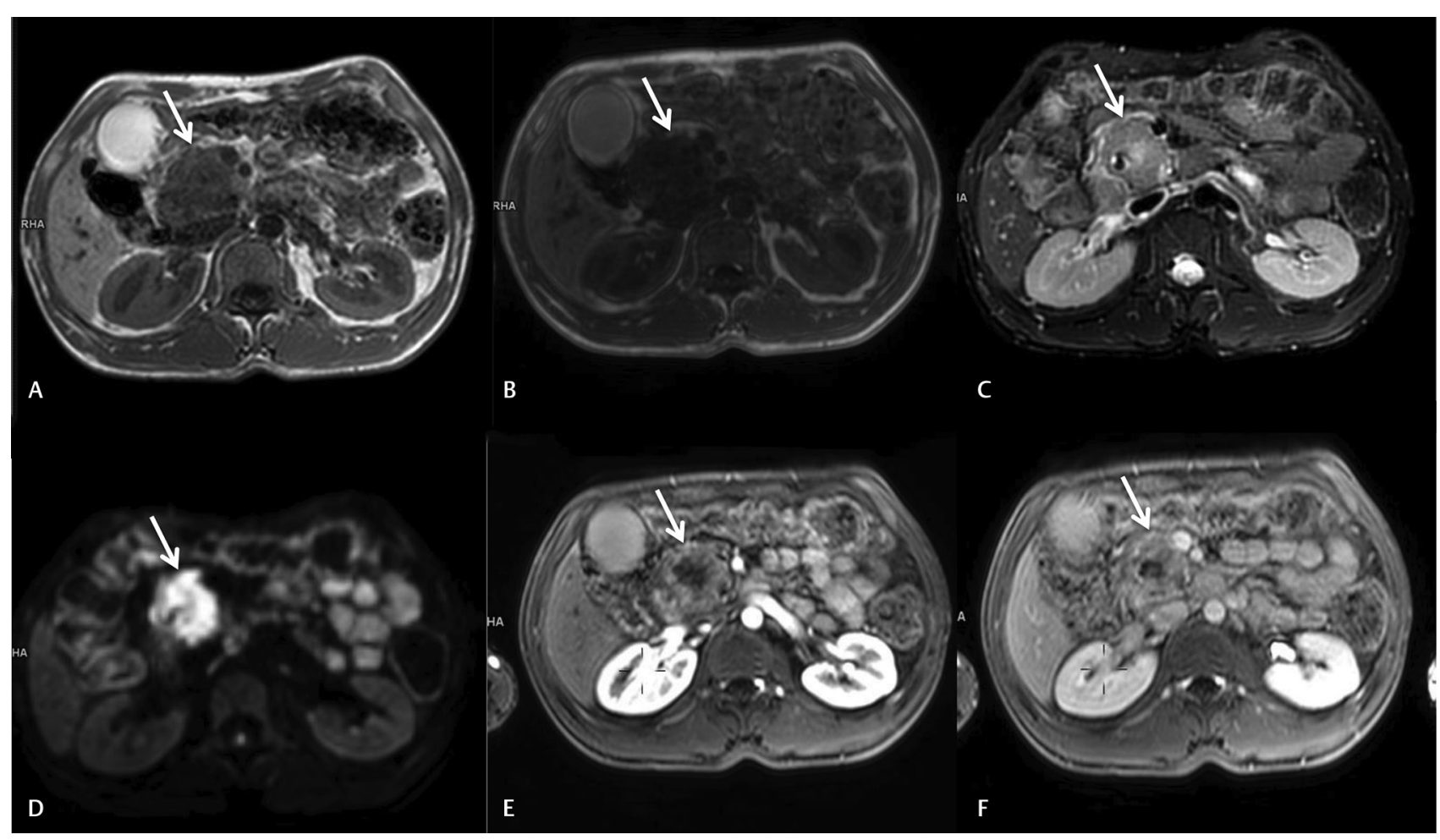

Fig. 3 (A-F) - shows a T1 hypointense (A, B - in and opposed phase), T2 heterogeneously hyperintense mass (C) in the head of pancreas showing no intracellular fat (A). The mass shows intense diffusion restriction (D) with heterogeneous arterial phase enhancement (E) sand washout on venous phase images (F). Histopathology confirmed the diagnosis of neuroendocrine tumor.

PET-CT offers improved spatial resolution with lower radiation dose than SPECT and hence is now the preferred imaging technique for detection of NETs. PET imaging requires labeling of agents with photon emitters like gallium (Ga)-68 and fluorine (F)-18. ${ }^{32}$ Somatostatin analogues developed for PET-CT include DOTA-tyrosine3-octreotide (DOTA-TOC) with affinity for somatostatin receptor 2 and DOTA-NaI-octreotide (DOTA-NOC), which binds to the receptor subtypes 2,3 , and 5 ( - Fig. 4). These two are labeled with Ga-68 for use. ${ }^{31}$ Fluorodeoxyglucose (18-FDG) is nonspecific and taken up by metabolically active tumors, and thus can be used for imaging poorly differentiated NETs. ${ }^{30}$ Hence a combined approach can be used for both detection and characterization of NETs. Poorly-differentiated NETs are negative on DOTA-NOC PET but show uptake on FDG PET. The sensitivity and specificity of PETCT has been reported to be $93 \%$ and $95 \%$, respectively. ${ }^{33}$ Another approach being used is targeting of metabolic pathways, by tagging amino acid precursors such as dihydroxyphenylalanine (DOPA) with F-18 and hydroxytryptophan (5-HTP) with carbon-11 (C-11). ${ }^{34}$ Efforts are also on to use the same concept for targeting these tumors using the approach of theranostics using peptide receptor radionuclide therapy (PRRT). Diagnostic SPECT/PET imaging agents (In-111/Ga-68 DOTATOC/-TATE) are tagged to therapeutic isotopes such as yttrium-90 (Y-90) and lutetium177 (Lu-177) and used to deliver targeted high-dose radiotherapy. ${ }^{10}$

\section{Contrast-Enhanced Ultrasound}

Intravenous ultrasound contrast agents are increasingly being used for diverse diseases. They are blood pool agents without a tissue phase and hence can give information about blood flow. Although approved by the United States Food and Drug Administration for cardiac imaging and evaluation of focal liver lesions, they are increasingly being

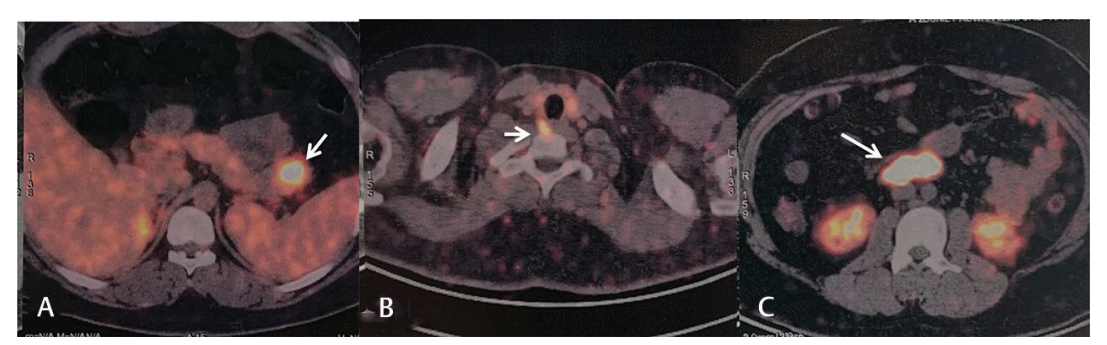

Fig. 4 Role of nuclear medicine in imaging patients with MEN-1 syndrome. (A) A mass in the pancreatic tail (arrow) showing uptake in 68Ga-NOTA-exendin-4 PET/CT suggesting presence of insulinoma. In the same patients, Ga-68 DOTANOC PET/CT showed other lesions in the right inferior parathyroid (B) as well as in the pancreatic uncinate $(\mathbf{C})$ which were resected. Final histopathology confirmed presence of parathyroid adenoma and gastrinoma in the uncinate process of pancreas. 


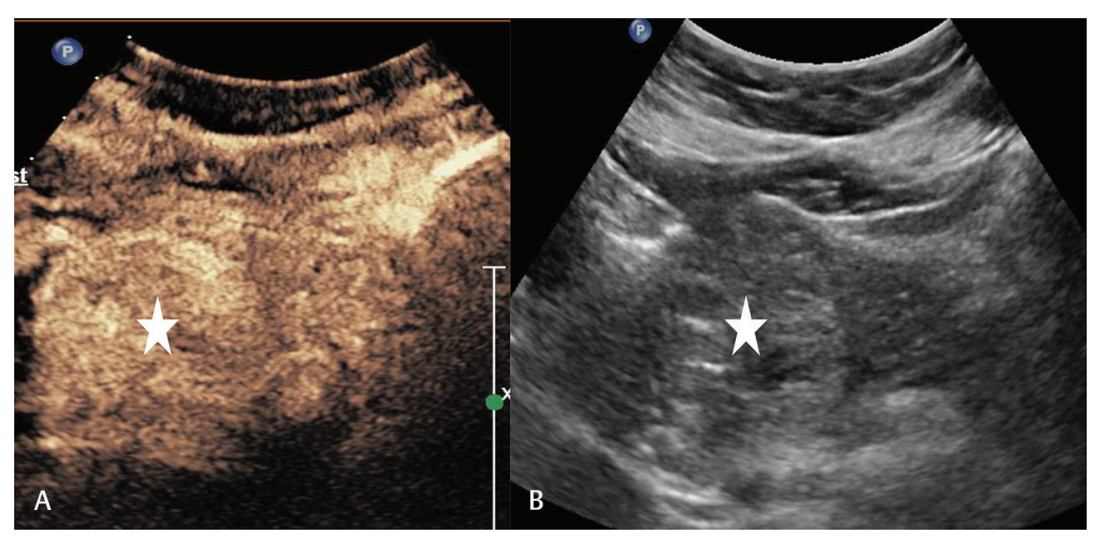

Fig. 5 Contrast-enhanced ultrasound (CEUS) image (A) showing intense uptake of contrast agent in the pancreatic head mass in early phase (asterisk). Corresponding gray scale image (B) shows a heterogeneous mass in the head of pancreas of mixed echogenicity which on resection was confirmed to be a non-functioning neuroendocrine tumor.

used for imaging other parts of the body due to their relative safety. Early studies have shown promising results with reported sensitivity of $87-89 \%$ for contrast-enhanced ultrasound (CEUS) compared with $24 \%$ for US alone in detection and localization of insulinomas ( - Fig. 5). ${ }^{35}$ Endoscopic Ultrasound

Sometimes, a lesion may be visualized on cross-sectional imaging but may not show typical features of NET. At times, the lesion may be too small or doubtful, producing just subtle contour bulge or slightly different attenuation/ signal intensity. These are the scenarios where endoscopic ultrasound (EUS) may come to the rescue. It has been reported to have a mean detection rate of around $90 \%$ and has the additional advantage of providing the opportunity to sample the lesion in the same sitting. ${ }^{36}$ The main disadvantage of EUS and EUS-guided fine needle aspiration cytology (FNAC) is that the technique is invasive and hence is associated with morbidity in $~ 1 \%$ cases. $^{37}$

\section{Imaging Findings}

For the purpose of discussion of imaging findings of NETs, they can broadly be divided into functional and nonfunctional tumors. Functional tumors usually present early on account of the symptoms they cause while nonfunctional tumors may stay clinically occult and reach large size before manifesting clinically. They usually present with pressure symptoms or may even be incidentally detected. Around $85 \%$ of pancreatic NETs are functional in nature. ${ }^{38}$

\section{Functional Pancreatic Neuroendocrine Tumors}

Functional pancreatic NETs manifest with systemic symptoms and are usually less than $2 \mathrm{~cm}$ at the time of imaging. ${ }^{16,20,39}$ Here the challenge is to detect the location of the tumor and map its spread. The most common functional pancreatic NETs are insulinomas and gastrinomas. ${ }^{10}$ Glucagonomas, somatostatinomas, and vasoactive intestinal peptide-secreting tumors (VIPomas) complete the list of functional pancreatic NETs.

\section{General Imaging Characteristics}

Functional NETs tend to be uniformly hypervascular and well-defined with intense arterial phase enhancement with washout on portal venous phase images.20,39 Though the sensitivity of arterial phase images is higher $(\sim 83-88 \%)$ than venous phase images ( 11-76\%), it is important to evaluate both these phases carefully since the lesions may be seen on either one of the phases..$^{10,18,20}$

Features like cystic change, heterogeneity, calcifications, and necrosis are generally seen in larger tumors but may uncommonly occur even in smaller tumors. ${ }^{40}$ Cystic tumors with a thin peripheral rim are seen in around 5 to $10 \%$ of cases. ${ }^{20}$

Though most functional neuroendocrine tumors share these imaging characteristics, it is imperative to discuss them individually to comprehensively understand their clinicoradiological presentation.

\section{Insulinomas}

The classical presentation of insulinomas is Whipple triad which consists of symptoms of hypoglycemia which are relieved after glucose ingestion and documented low glucose levels in blood. Insulinomas tend to be small, solitary, intrapancreatic lesions which are usually benign (in 90\% cases); $97 \%$ of insulinomas are intrapancreatic, $90 \%$ are solitary, and $40 \%$ are less than $1 \mathrm{~cm}$ in size. ${ }^{10}$ Tumor size of more than $3 \mathrm{~cm}$ should raise the suspicion of malignancy while multiplicity suggests syndromic association such as multiple endocrine neoplasia type 1 (MEN-1).39,41,42 Less than $10 \%$ of insulinomas are associated with MEN-1 syndrome. ${ }^{43}$ Insulinomas express somatostatin receptor type 3 and hence sensitivity of octreotide scan is limited ( 50\%) (octreotide binds to receptors 2 and 5). ${ }^{29,31,44} \mathrm{CT}$ and MRI are often more useful with good sensitivity ( - Fig. 6). ${ }^{10}$ However, the sensitivity of newer PET-CT techniques has been reported to be approximately 84 to $97 \%{ }^{45,46}$ A newer PET-CT metabolite, glucagon-like peptide- 1 receptor 68Ga-NOTA-exendin-4 PET/CT has been shown to have high sensitivity ( 99\%) for detection of insulinomas $\mathrm{s}^{46,47}$ ( Fig. 4). 


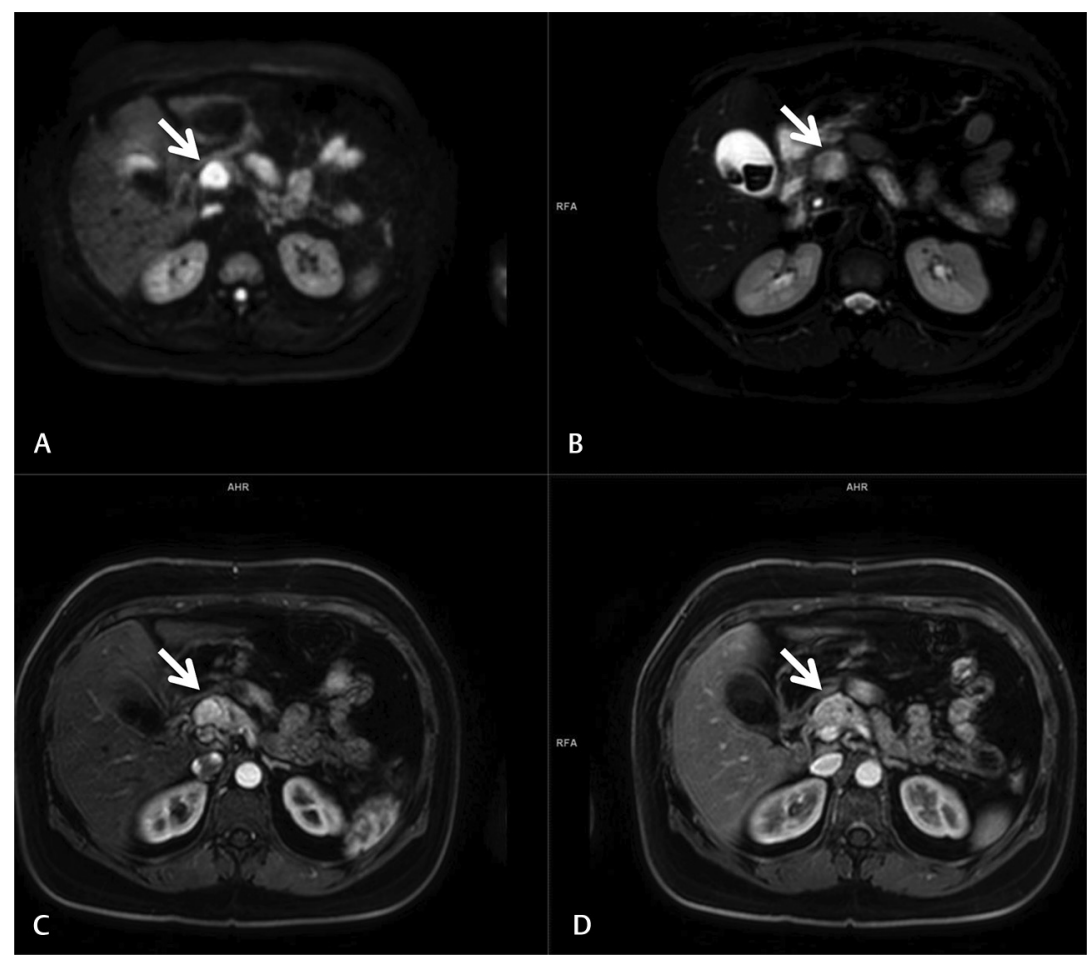

Fig. 6 (A-D) Presence of a T2 heterogeneously hyperintense mass in the head of pancreas showing intense diffusion restriction (A). The lesion shows arterial phase enhancement $(\mathbf{C})$ with washout in the venous phase images (D) consistent with a neuroendocrine tumor. Final histopathology confirmed the mass to be an insulinoma.

It is important to not only locally stage the tumor but also to look identify nodal and liver metastasis. ${ }^{39}$ The sensitivity and specificity of Ga-68 DOTANOC PET/CT is reported to be around $97 \%$ and $100 \%$ for detection of metastases. ${ }^{48}$ Though invasive techniques such as arterial stimulation and venous sampling, and transhepatic portal venous sampling have been conventionally used to improve localization, they are seldom used in current era owing to high sensitivity of various imaging modalities. Intraoperative US remains the gold standard for delimiting the lesion ${ }^{30,42,49}$ and is especially important when localized resections are planned.

\section{Gastrinomas}

Clinically, gastrinomas present with abdominal pain, secretory diarrhea, and hypercalcemia. Biochemically there is increased basal acid output, maximal acid output and serum gastrin levels. In contrast to insulinomas, gastrinomas are not exclusively intrapancreatic. More than a third of these tumors are located in extrapancreatic locations including the duodenum and peripancreatic lymph nodes ${ }^{10} ; 90 \%$ of gastrinomas are located in the gastrinoma triangle, bound by the junction of the cystic duct with the common bile duct superiorly; junction of the second and

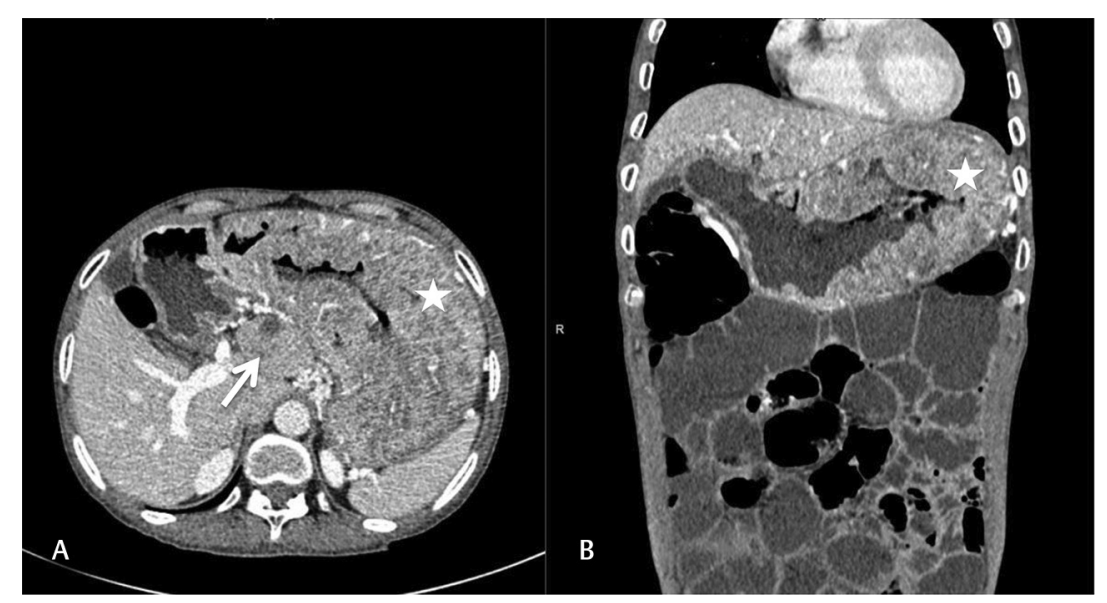

Fig. 7 (A, B) Presence of marked gastric rugal fold thickening predominantly in the fundus and proximal body of stomach (asterisk). A mass lesion was seen in the gastrohepatic location (arrow in $\mathbf{A}$ ) which on histopathology was consistent with a gastrinoma. 


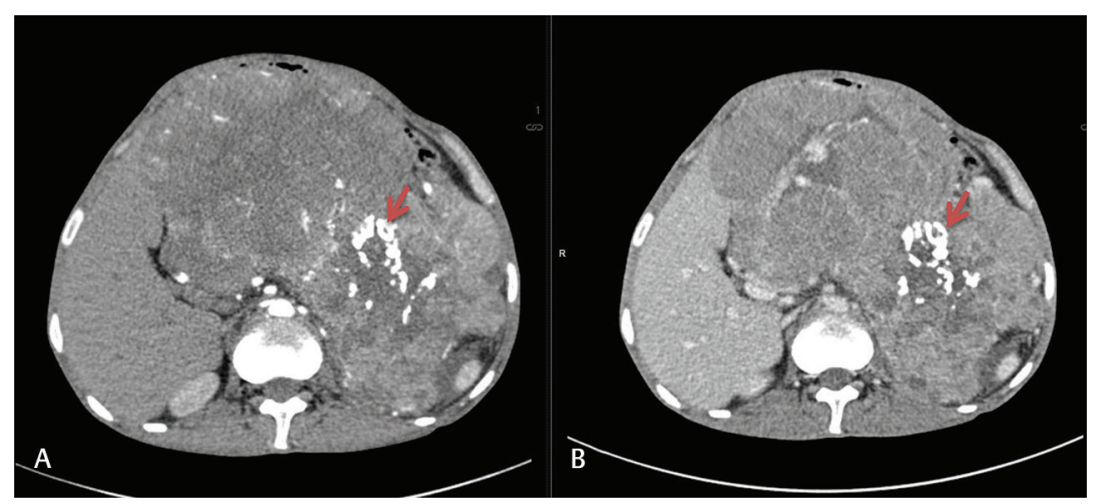

Fig. 8 Axial arterial (A) and venous phase (B) images showing a large heterogeneous mass in the pancreatic bed with chunky foci of calcification (arrows) which on histopathology was proven to be a poorly differentiated neuroendocrine tumor.

third part of duodenum inferolaterally, and the pancreatic neck medially. Less commonly, lesions may occur in the stomach and jejunum. ${ }^{20,39}$ Presence of gastric rugal thickening in association with hypervascular lesion in gastrinoma triangle suggests the diagnosis of gastrinoma ( - Fig. 7 ). Pancreatic gastrinomas usually occur in the head and tend to be larger lesions typically around 3 to $4 \mathrm{~cm}$ in size. ${ }^{50} \mathrm{In}$ contradistinction, duodenal lesions are typically subcentimetric and multiple. ${ }^{50}$ Hence, CT and MRI are typically better in detecting pancreatic lesions. Overall, EUS plays an important role in diagnosing gastrinomas and allows sampling of the lesion and or lymph nodes. ${ }^{20}$ Detection and staging may also be aided by octreotide scan or PET-CT as they express a high concentration of somatostatin receptors.

A higher percentage of gastrinomas ( $50-60 \%)$ are malignant at the time of diagnosis and that necessitates watchful inspection of locoregional nodes and the liver. ${ }^{20}$ About 15 to $35 \%$ of gastrinomas are associated with MEN-1 syndrome and thus gastrinomas are the commonest NET in this syndrome. ${ }^{43}$

\section{Glucagonomas}

After insulinomas and gastrinomas, glucagonomas are the third most common functional pancreatic NETs. The diagnosis is usually clinical with a diagnostic triad of hyperglycemia, necrolytic migratory erythema, and venous thrombosis. Serum glucagon levels more than $1,000 \mathrm{pg} / \mathrm{mL}$ are diagnostic. ${ }^{43}$ On cross-sectional imaging, these tend to be large tumors and are easily visible on imaging. Nuclear medicine scans are also helpful in localizing metastasis, if any.

\section{Other Functional Pancreatic NETs}

These are rare include VIPomas and somatostatinomas. VIPoma presents with Verner-Morrison syndrome, which is characterized by the clinical triad of watery diarrhea, hypokalemia, and achlorhydria. Serum levels of VIP more than $200 \mathrm{pg} / \mathrm{mL}$ are diagnostic. Somatostatin causes a decrease in the secretion of pancreatic enzymes, insulin, and cholecystokinin by the pancreas. Its oversecretion, thus, is clinically characterized by diarrhea, steatorrhea, diabetes, and gall stones. Most somatostatinomas are malignant and may have metastases at presentation. Fasting serum somatostatin levels above $100 \mathrm{pg} / \mathrm{mL}$ are diagnostic.

\section{Nonfunctioning Pancreatic Neuroendocrine Tumors}

Nonfunctioning pancreatic NETs present with symptoms secondary to mass effect, including abdominal pain, jaundice, and weight loss. They tend to be larger in size at presentation. ${ }^{20,40}$ Up to one-third of nonfunctional pancreatic NETs may be found incidentally. ${ }^{51}$ The term nonfunctioning is a misnomer because these tumors might secrete hormones such as pancreatic polypeptide, neurotensin, neuron-specific enolase, chromogranin A, $\alpha$ subunit of human chorionic gonadotropin, etc.. However, there is no clinical manifestation of these secretions. ${ }^{10}$ The larger size is associated with greater heterogeneity with areas of cystic change, necrosis, and foci of calcification ( $\mathbf{- F i g .} \mathbf{1})$. Some of these tumors may be predominantly cystic and may mimic solid pseudopapillary tumors. ${ }^{40}$ In addition, they are more likely to be metastatic at presentation $(60-80 \%)$ with lymph nodes and liver being common sites of metastasis. ${ }^{16,20}$ The tumor is more likely to be malignant if it contains foci of calcification (-Fig. 8). ${ }^{16}$ Neuroendocrine tumors may be associated with ductal dilation due to obstruction as well as can spread within the distended duct. ${ }^{16,20}$ About one-third of the nonfunctioning NETs may be associated with intravascular invasion manifesting as venous tumor thrombus. ${ }^{52}$ Multiple nonfunctional NETs may occur in MEN-1 and VHL syndrome or may occur sporadically (-Figs. 1, 9, 10). ${ }^{20}$

\section{Grading of Pancreatic Endocrine Tumors}

Grading of pancreatic neuroendocrine tumors is done using the ENETS/WHO grading system 2019..$^{53}$

Well-differentiated NETs are divided into 3 categories:

- Grade 1 (low grade): mitotic rate < 2 per 10 high-power fields and Ki67 rate $<3 \%$.

- Grade 2 (intermediate grade): mitotic rate 2 to 20 per 10 high-power fields or Ki67 rate 3 to $20 \%$.

- Grade 3 (high grade): mitotic rate $>20$ per 10 high-power fields or Ki67 rate $>20 \%$. 


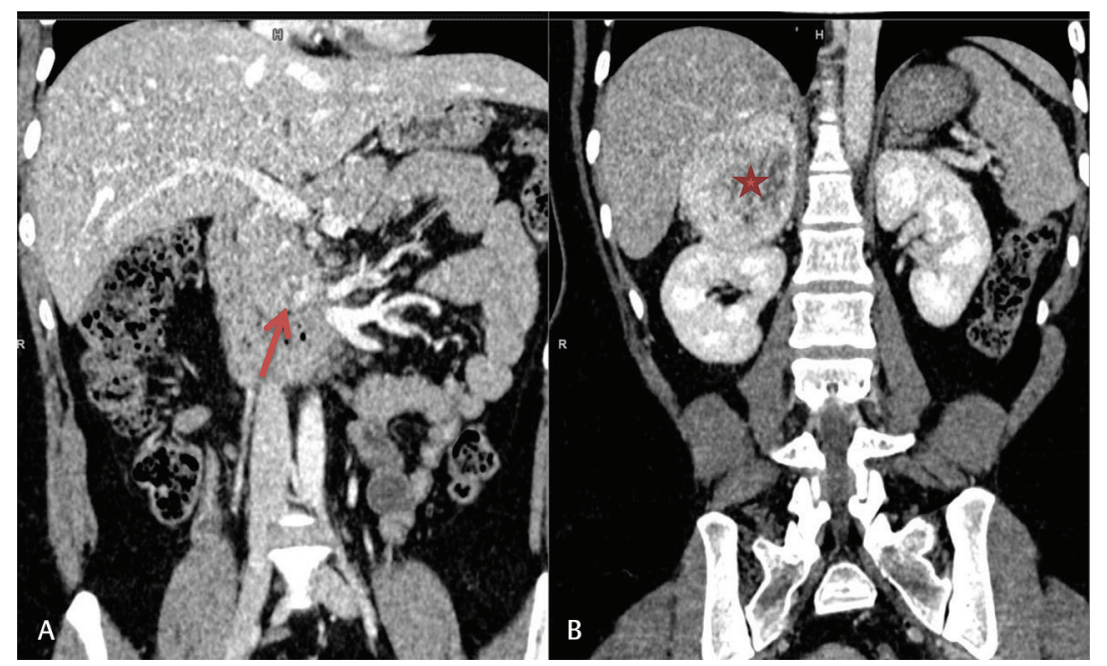

Fig. 9 Coronal CT images showing an enhancing nonfunctioning pancreatic neuroendocrine tumor (arrow in $\mathbf{A}$ ) and concomitant pheochromocytoma (asterisk in $\mathbf{B}$ ) in a patient of von Hippel-Lindau syndrome.

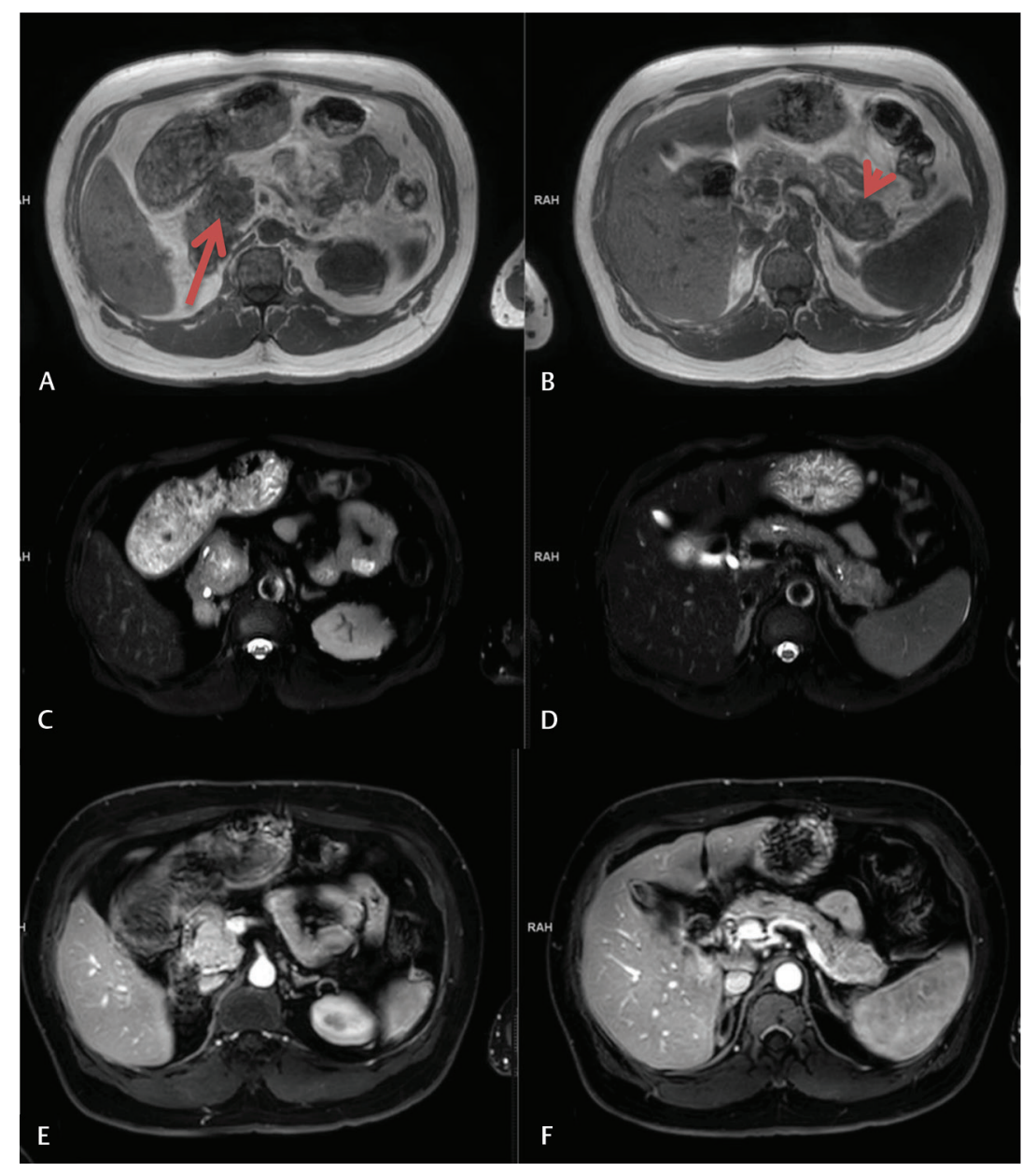

Fig. 10 (A-F) Two synchronous pancreatic neuroendocrine tumors in the uncinate (arrow) and tail of pancreas (arrowhead). The lesions were T1 hypointense (A, B), T2 hyperintense (C, D) with early enhancement (E, F). No syndromic association could be established.

Poorly differentiated NETs are termed neuroendocrine carcinomas and are high-grade lesions which can be small-cell type or large-cell type.
Poorly differentiated NETs portend a poorer prognosis for the patient and have a higher propensity for lymph nodal and/ or liver metastasis. They are dedifferentiated tumors which 
Table 2 Staging of pancreatic neuroendocrine tumors

\begin{tabular}{|l|l|l|}
\hline $\begin{array}{l}\text { AJCC } \\
\text { Stage }\end{array}$ & $\begin{array}{l}\text { Stage } \\
\text { grouping }\end{array}$ & Involvement \\
\hline I & T1N0M0 & $\begin{array}{l}\text { Tumor size }<2 \mathrm{~cm} \text {, confined to the pancreas. } \\
\text { No nodal/metastatic involvement }\end{array}$ \\
\hline II & T2N0M0 & $\begin{array}{l}\text { Tumor size } \geq 2 \mathrm{~cm},<4 \mathrm{~cm} \text {, confined to the } \\
\text { pancreas. No nodal/metastatic involvement }\end{array}$ \\
\cline { 2 - 4 } & T3N0M0 & $\begin{array}{l}\text { Tumor size }>4 \mathrm{~cm} \text {, confined to the pancreas } \\
\text { OR tumor involving the duodenum/ } \\
\text { common bile duct (CBD). No nodal/ } \\
\text { metastatic involvement }\end{array}$ \\
\hline III & T4N0M0 & $\begin{array}{l}\text { Tumor involving adjacent organs including } \\
\text { stomach/spleen/colon/adrenal OR tumor } \\
\text { involving celiac axis/superior mesenteric } \\
\text { artery. No nodal/metastatic deposit }\end{array}$ \\
\hline \multirow{2}{*}{ IV } & $\begin{array}{l}\text { Any T, } \\
\text { N1M0 }\end{array}$ & $\begin{array}{l}\text { Irrespective of tumor size and local invasion, } \\
\text { involvement of regional lymph nodes. No } \\
\text { metastatic deposit }\end{array}$ \\
\hline N, M1 Any & $\begin{array}{l}\text { Irrespective of local tumor spread or nodal } \\
\text { involvement, presence of distant metastasis }\end{array}$ \\
\hline \multicolumn{2}{|l}{}
\end{tabular}

express fewer somatostatin receptors and hence may be missed on octreotide studies. Due to their high metabolic rate, they are better visualized on FDG-PET studies. ${ }^{20,52,54}$ On imaging, these tumors show atypical enhancement patterns with decreased arterial enhancement and persistent or progressive enhancement of the tumor on venous phase imaging..$^{55} \mathrm{On}$ MR, these tumors show lower ADC values with low enhancement in the arterial phase. ${ }^{24}$ The presence of ill-defined margins, main pancreatic duct dilatation, vascular invasion, larger tumor size, and atypical enhancement pattern have been found to be associated with poorer histological grade ${ }^{56,57}$

\section{Staging}

The TNM staging AJCC UICC 8th edition is followed (-Table 2). ${ }^{58}$ Important points to note in the above-mentioned staging system are as follows:

1. Tumors confined to the pancreas include those extending into the adjacent fat.

2. Regional lymph nodes are defined differently for tumors of the head and for tumors involving pancreatic body and tail. For neuroendocrine tumors of the pancreatic head, regional lymph nodes include common bile duct, common hepatic artery, portal vein, posterior and anterior pancreatoduodenal arcades, superior mesenteric vein, and right lateral wall of superior mesenteric vein nodes. Common hepatic artery, celiac axis, splenic artery, and splenic hilar lymph nodes are considered locoregional lymph nodes for pancreatic body/tail neuroendocrine tumors.

3. Metastatic disease is further subclassified as M1ametastasis confined to the liver; M1b-involvement of extrahepatic sites such as lung, ovary, nonlocoregional lymph node, peritoneum, and bone, and M1c, when there is involvement of both hepatic and extrahepatic sites.

\section{Management}

The cornerstone of management of localized and resectable pancreatic neuroendocrine tumors is surgical resection. Adjuvant therapy after tumor resection does not have a proven role. Surgical treatment may be in the form of enucleation for small, well-defined tumors, resection of body/tail for distal tumors or pancreaticoduodenectomy/Whipple's procedure for tumors located in the pancreatic head. $38,43,59,60$

For metastatic disease, different treatment protocols have been tried. Surgical cytoreduction may aid in symptom reduction in functioning tumors as well as improve long-term survival. ${ }^{61}$ Other treatment modalities which have been tried for liver metastases include transarterial chemoembolization, radioembolization, radiofrequency ablation (RFA), cryoablation, and percutaneous alcohol ablation. ${ }^{62-64}$ No systematic reviews are available comparable these techniques; however, surgery is considered the standard approach for resectable liver lesions. If the lesions are not completely resectable, then RFA or other techniques mentioned above may be used for palliation of symptoms. If the lesions cannot be subjected to any of these, somatostatin analogs such as octreotide, octreotate, lanreotide, or edotreotide can be radiolabeled with 111 indium, 90 ytrium, or 177 lutetium for targeted radiotherapy. ${ }^{65,66}$

\section{Conclusion}

Imaging plays an important role in detection, accurate characterization, and extent delineation of pancreatic neuroendocrine tumors. Future research these days is directed toward imaging prognostication and therapeutic response assessment.

\section{Conflict of Interest}

None declared.

\section{References}

1 Fraenkel M, Kim MK, Faggiano A, Valk GD. Epidemiology of gastroenteropancreatic neuroendocrine tumours. Best Pract Res Clin Gastroenterol 2012;26(6):691-703

2 Lawrence B, Gustafsson BI, Chan A, Svejda B, Kidd M, Modlin IM. The epidemiology of gastroenteropancreatic neuroendocrine tumors. Endocrinol Metab Clin North Am 2011;40(1):1-18,vii vii

3 Zhou J, Enewold L, Stojadinovic A, et al. Incidence rates of exocrine and endocrine pancreatic cancers in the United States. Cancer Causes Control 2010;21(6):853-861

4 Edge SB, Byrd DR, Compton CC, Fritz AG, Greene FL, Trotti A, eds. Exocrine and endocrine pancreas. In: AJCC Cancer Staging Manual. 7th ed. New York, NY: Springer; 2010: 241-249

5 Amin MB, Edge SB, Greene FL, et al. eds. Exocrine and endocrine pancreas. In: AJCC Cancer Staging Manual. 7th ed. New York, NY: Springer; 2010 241-249

6 Baur AD, Pavel M, Prasad V, Denecke T. Diagnostic imaging of pancreatic neuroendocrine neoplasms (pNEN): tumor detection, staging, prognosis, and response to treatment. Acta Radiol 2016;57(3):260-270 
7 Jensen RT, Berna MJ, Bingham DB, Norton JA. Inherited pancreatic endocrine tumor syndromes: advances in molecular pathogenesis, diagnosis, management, and controversies. Cancer 2008;113(7, Suppl):1807-1843

8 Kulke MH, Siu LL, Tepper JE, et al. Future directions in the treatment of neuroendocrine tumors: consensus report of the National Cancer Institute Neuroendocrine Tumor clinical trials planning meeting. J Clin Oncol 2011;29(7):934-943

9 Hilal T. Current understanding and approach to well differentiated lung neuroendocrine tumors: an update on classification and management. Ther Adv Med Oncol 2017;9(3):189-199

10 Tamm EP, Bhosale P, Lee JH, Rohren EM. State-of-the-art imaging of pancreatic neuroendocrine tumors. Surg Oncol Clin N Am 2016;25(2):375-400

11 Wang YW, Zhang XH, Wang BT, et al. Value of texture analysis of intravoxel incoherent motion parameters in differential diagnosis of pancreatic neuroendocrine tumor and pancreatic adenocarcinoma. Chin Med Sci J 2019;34(1):1-9

12 Hwang EJ, Lee JM, Yoon JH, et al. Intravoxel incoherent motion diffusion-weighted imaging of pancreatic neuroendocrine tumors: prediction of the histologic grade using pure diffusion coefficient and tumor size. Invest Radiol 2014;49(6):396-402

13 Zhu L, Xue H, Sun H, et al. Insulinoma detection with MDCT: is there a role for whole-pancreas perfusion? AJR Am J Roentgenol 2017;208(2):306-314

14 Sundin A, Vullierme MP, Kaltsas G, Plöckinger U; Mallorca Consensus Conference participants; European Neuroendocrine Tumor Society. ENETS Consensus Guidelines for the Standards of Care in Neuroendocrine Tumors: radiological examinations. Neuroendocrinology 2009;90(2):167-183

15 Tan $\mathrm{EH}$, Tan $\mathrm{CH}$. Imaging of gastroenteropancreatic neuroendocrine tumors. World J Clin Oncol 2011;2(1):28-43

16 Sahani DV, Bonaffini PA, Fernández-Del Castillo C, Blake MA. Gastroenteropancreatic neuroendocrine tumors: role of imaging in diagnosis and management. Radiology 2013;266(1):38-61

17 Dogeas E, Chong CC, Weiss MJ, Ahuja N, Choti MA. Can echogenic appearance of neuroendocrine liver metastases on intraoperative ultrasonography predict tumor biology and prognosis? HPB (Oxford) 2018;20(3):237-243

18 Fidler JL, Fletcher JG, Reading CC, et al. Preoperative detection of pancreatic insulinomas on multiphasic helical CT. AJR Am J Roentgenol 2003;181(3):775-780

19 Gouya H, Vignaux O, Augui J, et al. CT, endoscopic sonography, and a combined protocol for preoperative evaluation of pancreatic insulinomas. AJR Am J Roentgenol 2003;181(4):987-992

20 Lewis RB, Lattin GEJr,PaalE.Pancreaticendocrinetumors:radiologic-clinicopathologic correlation. Radiographics 2010;30(6): $1445-1464$

21 Rockall AG, Reznek RH. Imaging of neuroendocrine tumours (CT/MR/US) Best Pract Res Clin Endocrinol Metab 2007; 21(1):43-68

22 Lin XZ, Wu ZY, Tao R, et al. Dual energy spectral CT imaging of insulinoma-Value in preoperative diagnosis compared with conventional multi-detector CT. Eur J Radiol 2012;81(10):2487-2494

23 Brenner R, Metens T, Bali M, Demetter P, Matos C. Pancreatic neuroendocrine tumor: added value of fusion of T2-weighted imaging and high b-value diffusion-weighted imaging for tumor detection. Eur J Radiol 2012;81(5):e746-e749

24 Jang KM, Kim SH, Song KD, Kim YK, Lee SJ, Choi D. Differentiation of solid-type serous cystic neoplasm from neuroendocrine tumour in the pancreas: value of abdominal MRI with diffusion-weighted imaging in comparison with MDCT. Clin Radiol 2015;70(2):153-160

25 Kang BK, Kim JH, Byun JH, et al. Diffusion-weighted MRI: usefulness for differentiating intrapancreatic accessory spleen and small hypervascular neuroendocrine tumor of the pancreas. Acta Radiol 2014;55(10):1157-1165
26 Jang KM, Kim SH, Min JH, et al. Value of diffusion-weighted MRI for differentiating malignant from benign intraductal papillary mucinous neoplasms of the pancreas. AJR Am J Roentgenol 2014;203(5):992-1000

27 Guo C-G, Ren S, Chen X, et al. Pancreatic neuroendocrine tumor: prediction of the tumor grade using magnetic resonance imaging findings and texture analysis with 3-T magnetic resonance. Cancer Manag Res 2019;11:1933-1944

28 Guo C, Chen X, Xiao W, Wang Q, Sun K, Wang Z. Pancreatic neuroendocrine neoplasms at magnetic resonance imaging: comparison between grade 3 and grade 1/2 tumors. OncoTargets Ther 2017; 10:1465-1474

29 Tamm EP, Kim EE, Ng CS. Imaging of neuroendocrine tumors. Hematol Oncol Clin North Am 2007;21(3):409-432,vii

30 Balachandran A, Bhosale PR, Charnsangavej C, Tamm EP. Imaging of pancreatic neoplasms. Surg Oncol Clin N Am 2014;23(4):751-788

31 Rufini V, Calcagni ML, Baum RP. Imaging of neuroendocrine tumors. Semin Nucl Med 2006;36(3):228-247

32 Hofman MS, Lau WF, Hicks RJ. Somatostatin receptor imaging with 68Ga DOTATATE PET/CT: clinical utility, normal patterns, pearls, and pitfalls in interpretation. Radiographics 2015;35(2):500-516

33 Geijer H, Breimer LH. Somatostatin receptor PET/ CT in neuroendocrine tumours: update on systematic review and meta-analysis. Eur J Nucl Med Mol Imaging 2013;40(11):1770-1780

34 Kauhanen S, Seppänen M, Minn H, Nuutila P. Clinical PET imaging of insulinoma and beta-cell hyperplasia. Curr Pharm Des 2010;16(14):1550-1560

35 An L, Li W, Yao KC, et al. Assessment of contrast-enhanced ultrasonography in diagnosis and preoperative localization of insulinoma. Eur J Radiol 2011;80(3):675-680

36 Ichikawa T, Peterson MS, Federle MP, et al. Islet cell tumor of the pancreas: biphasic CT versus MR imaging in tumor detection. Radiology 2000;216(1):163-171

37 Wang KX, Ben QW, Jin ZD, et al. Assessment of morbidity and mortality associated with EUS-guided FNA: a systematic review. Gastrointest Endosc 2011;73(2):283-290

38 Hochwald SN, Zee S, Conlon KC, et al. Prognostic factors in pancreatic endocrine neoplasms: an analysis of 136 cases with a proposal for low-grade and intermediate-grade groups. J Clin Oncol 2002;20(11):2633-2642

39 Heller MT, Shah AB. Imaging of neuroendocrine tumors. Radiol Clin North Am 2011;49(3):529-548,vii

40 Buetow PC, Miller DL, Parrino TV, Buck JL. Islet cell tumors of the pancreas: clinical, radiologic, and pathologic correlation in diagnosis and localization. Radiographics 1997;17(2):453-472, quiz472A-472B

41 Ectors N. Pancreatic endocrine tumors: diagnostic pitfalls. Hepatogastroenterology 1999;46(26):679-690

42 Mansour JC, Chen H. Pancreatic endocrine tumors. J Surg Res 2004;120(1):139-161

43 Davies K, Conlon KC. Neuroendocrine tumors of the pancreas. Curr Gastroenterol Rep 2009;11(2):119-127

44 Druce MR, Muthuppalaniappan VM, O'Leary B, et al. Diagnosis and localisation of insulinoma: the value of modern magnetic resonance imaging in conjunction with calcium stimulation catheterisation. Eur J Endocrinol 2010;162(5):971-978

45 Prasad V, Sainz-Esteban A, Arsenic R, et al. Role of (68)Ga somatostatin receptor PET/CT in the detection of endogenous hyperinsulinaemic focus: an explorative study. Eur J Nucl Med Mol Imaging 2016;43(9):1593-1600

46 Luo Y, Pan Q, Yao S, et al. Glucagon-like peptide-1 receptor PET/CT with 68Ga-NOTA-exendin-4 for detecting localized insulinoma: a prospective cohort study. J Nucl Med 2016;57(5):715-720 
47 Luo Y, Yao S, Li F. 68Ga-exendin-4 PET/CT is both sensitive and specific in diagnosing insulinomas. J Nucl Med 2017;58(supplement 1):237

48 Naswa N, Sharma P, Kumar A, et al. Gallium-68-DOTA-NOC PET/CT of patients with gastroenteropancreatic neuroendocrine tumors: a prospective single-center study. AJR Am J Roentgenol 2011;197(5):1221-1228

49 Brown CK, Bartlett DL, Doppman JL, et al. Intraarterial calcium stimulation and intraoperative ultrasonography in the localization and resection of insulinomas. Surgery 1997;122(6):1189-1193,discussion1193-1194

50 Horton KM, Hruban RH, Yeo C, Fishman EK. Multi-detector row CT of pancreatic islet cell tumors. Radiographics 2006;26(2):453-464

51 Gullo L, Migliori M, Falconi M, et al. Nonfunctioning pancreatic endocrine tumors: a multicenter clinical study. Am J Gastroenterol 2003;98(11):2435-2439

52 Balachandran A, Tamm EP, Bhosale PR, et al. Venous tumor thrombus in nonfunctional pancreatic neuroendocrine tumors. AJR Am J Roentgenol 2012;199(3):602-608

53 Klimstra DS, Kloppell G, La Rosa S, Rindi G, Classification of neuroendocrine neoplasms of the digestive system. In: WHO Classification of Tumours: Digestive System Tumours. 5th ed. Lyon, France: WHO Classification of Tumours Editorial Board (Ed), International Agency for Research on Cancer; 2019:16

54 Binderup T, Knigge U, Loft A, Federspiel B, Kjaer A. 18F-fluorodeoxyglucose positron emission tomography predicts survival of patients with neuroendocrine tumors. Clin Cancer Res 2010;16(3):978-985

55 Cappelli C, Boggi U, Mazzeo S, et al. Contrast enhancement pattern on multidetector CT predicts malignancy in pancreatic endocrine tumours. Eur Radiol 2015;25(3):751-759

56 Luo Y, Dong Z, Chen J, et al. Pancreatic neuroendocrine tumours: correlation between MSCT features and pathological classification. Eur Radiol 2014;24(11):2945-2952
57 Takumi K, Fukukura Y, Higashi M, et al. Pancreatic neuroendocrine tumors: correlation between the contrast-enhanced computed tomography features and the pathological tumor grade. Eur J Radiol 2015;84(8):1436-1443

58 Bergsland EKWE, Rindo G, Neuroendocrine tumors of the pancreas. In: Amin MB, ed. AJCC Cancer Staging Manual, 8th ed. Chicago, IL American Joint Committee on Cancer 2017:407

59 Kazanjian KK, Reber HA, Hines OJ. Resection of pancreatic neuroendocrine tumors: results of 70 cases. Arch Surg 2006;141(8):765-769,discussion769-770

60 Phan GQ, Yeo CJ, Hruban RH, Littemoe KD, Pitt HA, Cameron JL. Surgical experience with pancreatic and peripancreatic neuroendocrine tumors: review of 125 patients. J Gastrointest Surg 1998;2(5):473-482

61 Sarmiento JM, Que FG. Hepatic surgery for metastases from neuroendocrine tumors. Surg Oncol Clin N Am 2003;12(1):231-242

62 Gupta S, Johnson MM, Murthy R, et al. Hepatic arterial embolization and chemoembolization for the treatment of patients with metastatic neuroendocrine tumors: variables affecting response rates and survival. Cancer 2005;104(8): 1590-1602

63 King J, Quinn R, Glenn DM, et al. Radioembolization with selective internal radiation microspheres for neuroendocrine liver metastases. Cancer 2008;113(5):921-929

64 Siperstein AE, Berber E. Cryoablation, percutaneous alcohol injection, and radiofrequency ablation for treatment of neuroendocrine liver metastases. World J Surg 2001;25(6):693-696

65 Bushnell DL Jr, O'Dorisio TM, O'Dorisio MS, et al. 90Y-edotreotide for metastatic carcinoid refractory to octreotide. J Clin Oncol 2010;28(10):1652-1659

66 Teunissen JJ, Kwekkeboom DJ, de Jong M, Esser JP, Valkema R, Krenning EP. Endocrine tumours of the gastrointestinal tract. Peptide receptor radionuclide therapy. Best Pract Res Clin Gastroenterol 2005;19(4):595-616 